\title{
Autophagy activated by silibinin contributes to glioma cell death via induction of oxidative stress- mediated BNIP3-dependent nuclear translocation of AIF
}

\author{
Chongcheng Wang ${ }^{1,2}$, Chuan He ${ }^{1,2}$, Shan Lu ${ }^{1,2}$, Xuanzhong Wang ${ }^{1,2}$, Lei Wang ${ }^{1,2}$, Shipeng Liang ${ }^{1,2}$, Xinyu Wang ${ }^{3}$,
} Meihua Piao ${ }^{4}$, Jiayue Cui ${ }^{5}$, Guangfan $\mathrm{Chi}^{6}$ and Pengfei Ge (B),

\begin{abstract}
Induction of lethal autophagy has become a strategy to eliminate glioma cells, but it remains elusive whether autophagy contributes to cell death via causing mitochondria damage and nuclear translocation of apoptosis inducing factor (AIF). In this study, we find that silibinin induces AIF translocation from mitochondria to nuclei in glioma cells in vitro and in vivo, which is accompanied with autophagy activation. In vitro studies reveal that blocking autophagy with 3MA, bafilomycin A1 or by knocking down ATG5 with SiRNA inhibits silibinin-induced mitochondrial accumulation of superoxide, AIF translocation from mitochondria to nuclei and glioma cell death. Mechanistically, silibinin activates autophagy through depleting ATP by suppressing glycolysis. Then, autophagy improves intracellular $\mathrm{H}_{2} \mathrm{O}_{2}$ via promoting p53-mediated depletion of GSH and cysteine and downregulation of $x C T$. The increased $\mathrm{H}_{2} \mathrm{O}_{2}$ promotes silibinin-induced BNIP3 upregulation and translocation to mitochondria. Knockdown of BNIP3 with SiRNA inhibits silibinin-induced mitochondrial depolarization, accumulation of mitochondrial superoxide, and AIF translocation from mitochondria to nuclei, as well as prevents glioma cell death. Furthermore, we find that the improved $\mathrm{H}_{2} \mathrm{O}_{2}$ reinforces silibinin-induced glycolysis dysfunction. Collectively, autophagy contributes to silibinininduced glioma cell death via promotion of oxidative stress-mediated BNIP3-dependent nuclear translocation of AIF.
\end{abstract}

\section{Introduction}

Glioma is an aggressive malignant brain tumor and constitutes the major causes leading to death in both pediatric and adult populations ${ }^{1}$. The median survival time of the patients with newly diagnosed glioma is only 14.6 months, despite these patients are treated with surgical removal of tumor followed by radiotherapy and chemotherapy with temozolomide $(\mathrm{TMZ})^{2}$. As an alkylating agent, TMZ is widely used for treating primary and

\footnotetext{
Correspondence: Pengfei Ge (gepf@jlu.edu.cn)

'Department of Neurosurgery, First Hospital of Jilin University, 130021

Changchun, China

${ }^{2}$ Research Center of Neuroscience, First Hospital of Jilin University, 130021

Changchun, China

Full list of author information is available at the end of the article

Edited by G.M. Fimia
}

recurrent high-grade gliomas, but its efficacy is often limited by development of resistance ${ }^{3}$. Therefore, new medicines are needed for glioma treatment.

Autophagy that is characterized morphologically with formation of autophagosomes or autolysosomes in cytoplasm is a degradation pathway by which intracellular materials or impaired organelles are delivered to lysosomes for clearance ${ }^{4}$. Autophagy plays dual roles in regulation of cell destiny. On the one hand, it protects cells against detrimental stresses. On the other hand, it contributes to cell death, which is designated as autophagic death ${ }^{4}$. It was found that autophagy protected neuroblastoma cells by removing misfolded proteins, which were generated during the process of oxidative stress $^{5}$. Autophagy was also reported to abrogate 
cisplatin-induced death in hepatocellular carcinoma cells via inhibiting accumulation of reactive oxygen species (ROS) by clearing damaged mitochondria ${ }^{6}$. Thus, the protection of autophagy against cell damage is closely associated with suppression of oxidative stress. In the case of autophagic death, autophagy is also accompanied with accumulation of intracellular ROS and generally thought to be activated by $\operatorname{ROS}^{7,8}$. However, it remains elusive whether autophagy could promote cell death via improving intracellular ROS.

Mitophagy refers to selective removal of damaged mitochondria via autophagy machinery ${ }^{9}$. Although mitophagy rescues cell death via clearing injured mitochondria $^{6}$, accumulating evidence showed that excessive or sustained mitophagy could also lead to cell death ${ }^{10,11}$. It was reported that ceramide triggered glioma cell death via overactivation of mitophagy ${ }^{10}$. Moreover, mitophagy was found to contribute to the glioma cell death induced by chemical compound AT $101^{11}$. Given that only damaged mitochondria could be recognized and degraded via autophagy pathway ${ }^{9}$, it remains elusive why excessive clearance of damaged mitochondria by autopahgy results in cell death. This makes us speculate that overactivated autophagy might play a role in causing mitochondrial damage.

Silibinin is a biologically active component of silymarin that is a polyphenolic extract from milk thistle (Silybum marianum) (Fig. 1a). It has been widely used to treat and prevent many types of hepatobiliary disorders including alcoholic liver disease, nonalcoholic fatty liver disease, and mushroom poisoning ${ }^{12,13}$. Moreover, silibinin not only exert potent inhibitory effect on various types of cancer cells, such as breast cancer cells, prostate cancer cells, and glioma cells ${ }^{7,14,15}$, but also sensitizes glioma cells to TMZ, TRAIL or arsenic trioxide treatment and prevents hepatocyte injury induced by chemotherapy agent cisplatin $^{16-19}$. Although silibinin could induce autophagic death in cancer cells ${ }^{7,8}$, the biochemical events downstream silibinin-induced autophagy remains unclear. Therefore, in this study, we used glioma cell lines and nude mice with xenografted glioma to investigate whether autophagy plays a role in promoting mitochondria damage and its potential mechanism.

\section{Materials and methods Reagents}

Silibinin was purchased from MedChemExpress (Monmouth Junction, NJ). Glutathione (GSH) was from Sigma (St.Louis, MO). Silibinin was dissolved in DMSO to a storage concentration of $200 \mathrm{mmol} / \mathrm{L}$. GSK-872 was from Calbiochem company (Billerica, MA, USA). GKT137831, necrostatin-1(Nec-1), NSA (Necrosulfonamide), and ferrostatin-1(Fer-1) were from selleckchem Company (Houston, TX). Deferoxamine was from Abcam company (Cambridge, MA). Anti-AIF (\#4642) and phospho-p53(Ser15) (\#9284) antibody were from cell signaling company (Danvers, MA). The primary antibodies against the following proteins ATG5(ab109490), LC3B(192890) p62/SQSTM1(ab56416), $\quad$ xCT(ab175186), BNIP3 (ab10433), PKM2(ab150377), HK II(ab209847), PFKP (ab119796), HIF-1 $\alpha$ (ab51608), GPX4(ab125066), p53 (ab26), TOMM20(ab186735), H2A(ab177308), and $\beta$-Actin (ab8226) were all purchased from Abcam company (Cambridge, MA). Other reagents were purchased from Sigma (St. Louis, MO).

\section{Cell line and culture}

Human U87, U251, SHG-44, and rat C6 glioma cells were all obtained from Shanghai Institute of Cell Biology, Chinese Academy of Sciences (Shanghai, China). The cells were cultured in DMEM supplemented with $10 \%$ fetal bovine serum, $2 \mathrm{mmol} / \mathrm{L}$ glutamine, penicillin $(100 \mathrm{U} / \mathrm{mL})$ and streptomycin $(100 \mu \mathrm{g} / \mathrm{mL})$, and maintained at $37^{\circ} \mathrm{C}$ and $5 \% \mathrm{CO}_{2}$ in a humid environment. Cells in the mid-log phase were used in the experiments.

\section{Cellular viability and cell death assays}

U87 $\left(5 \times 10^{4}\right.$ cells/well $)$, U251 $\left(5 \times 10^{4}\right.$ cells/well $)$, SHG$44\left(5 \times 10^{4}\right.$ cells/well $)$, and C6 $\left(1 \times 10^{5}\right.$ cells/well $)$ glioma cells were treated with target compounds after being seeded onto 96-well microplate with six duplicated wells and cultured $24 \mathrm{~h}$. Cellular viability was assessed using an MTT assay, and was expressed as a ratio to the absorbance value at $570 \mathrm{~nm}$ of the control cells. Cell death was assessed by using lactate dehydrogenase cytotoxicity assay kit according to the manufacturer's instructions (Beyotime Biotech, Nanjing, China), the absorbance value of each sample was read at $490 \mathrm{~nm}$, and cell death ratio was calculated by using the following formula: cell death ratio $\%=(\mathrm{A}$ sample $-\mathrm{A}$ control $/ \mathrm{A} \max -\mathrm{A}$ control $) \times 100$. A sample: sample absorbance value; A control: the absorbance value of control group; A max: the absorbance value of positive group.

\section{Measurement of mitochondrial membrane potential and mitochondrial superoxide}

Mitochondrial membrane potential was assayed by using JC-1 staining (Beyotime Biotech, Nanjing, China) as described by manufacture's instruction. The collected cells were analyzed by flow cytometry (FACScan, Becton Dickinson, San Jose, CA). Mitochondrial superoxide was assayed by using MitoSOX red according to manufacturer's description (Invitrogen, Eugene, OR). The red fluorescence density was measured at an excitation wavelength of $510 \mathrm{~nm}$ and an emission wavelength at $580 \mathrm{~nm}$, and was expressed as a ratio to the fluorescence in control cells. The cells seeded on a six-well plate were treated as the same as above described, and then observed 


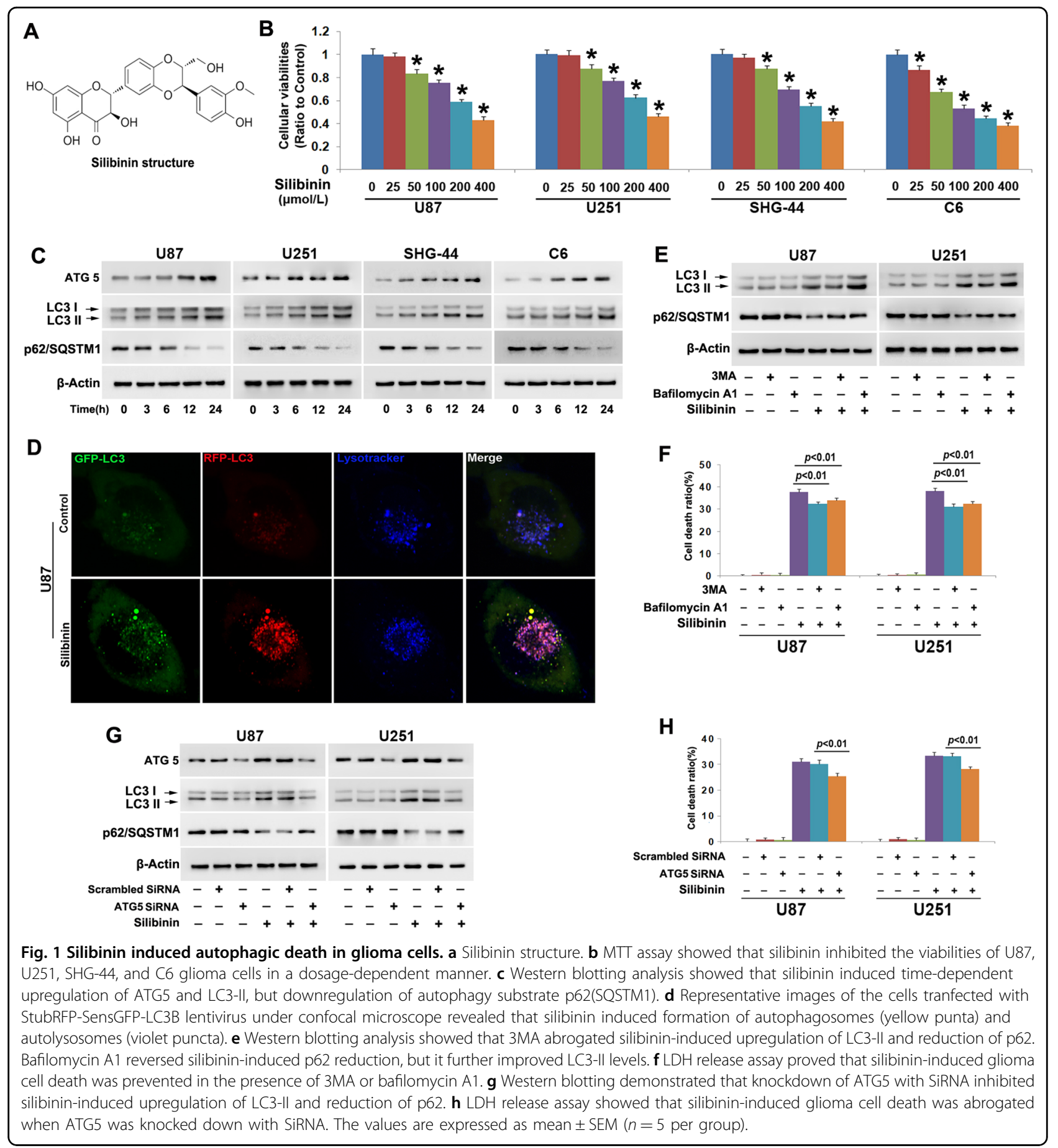

under fluorescence microscope (Olympus IX71, Tokyo, Japan). All the measurements and observation were performed by a researcher who was blinded to group allocation and repeated for five times.

\section{Measurement of intracellular $\mathrm{H}_{2} \mathrm{O}_{2}$ content}

The content of intracellular $\mathrm{H}_{2} \mathrm{O}_{2}$ in treated glioma cells was analyzed with a $\mathrm{H}_{2} \mathrm{O}_{2}$ assay kit (Beyotime Institute of
Biotechnology, Nanjing, China) according to the manufacturer protocol. In brief, the cells were collected by centrifugation at $800 \times g$ for $5 \mathrm{~min}$ and washed twice with PBS. The cell pellets and $10 \mathrm{mg}$ xenograft glioma tissues were lysed in lysis buffer by repeated cycles of freezing and thawing under liquid nitrogen and centrifuged at $12,000 \times g$ for $5 \mathrm{~min}$. Then, $50 \mathrm{~mL}$ of supernatants and $100 \mathrm{~mL}$ of test solution were added into a tube, placed at 
room temperature for $30 \mathrm{~min}$, and measured immediately with a spectrophotometer at a wavelength of $560 \mathrm{~nm}$. Absorbance values were calibrated to a standard concentration curve to calculate the concentration of $\mathrm{H}_{2} \mathrm{O}_{2}$. The measurement was performed by a researcher who was blinded to group allocation and repeated for five times. Finally, the results were expressed as a ratio to the concentration of the control cells.

\section{Transfection of small interfering RNA (SiRNA)}

The cells were seeded onto a culture dish. Transfection of siRNA was performed by using Lipofectamine 2000 (Invitrogen, Eugene, OR) according to the manufacturer's instructions.

ATG5 SiRNA (5'-GACGUUGGUAACUGACAAATT-3'), BNIP3 SiRNA (5'- GAUUACUUCUGAGCUUGCATT$\left.3^{\prime}\right)$,

AIF SiRNA (5'-GCAGUGGCAAGUUACUUAUTT- $3^{\prime}$ ) and scrambled SiRNA (5'-UUCUCCGAACGUGUC ACGUTT-3') were all purchased from GenePharma Company (Suzhou, China). After SiRNA transfection overnight, the cells were incubated with silibinin at indicated dosage for subsequent experiments.

\section{Rat C6 tumor xenograft in mice}

The female athymic BALB/c nude mice (age 4 weeks, weight $20-22 \mathrm{~g}$, Beijing Vital River laboratory animal technology company, China) were housed in a specific pathogen-free environment under the condition of 12-h light/12-h dark cycle, free access to food and water, and acclimatized to their surroundings for 3 days. The mice were cared in accordance with the guidelines for experimental animals of Jilin University and the study was approved by the ethics committee of First Hospital of Jilin University (Changchun, China). The animal quantity used in this study was estimated as described previously ${ }^{20}$. A total of $1 \times 10^{7}$ logarithmically growing C6 cells in $100 \mu \mathrm{L}$ of PBS were subcutaneously injected into the right flank of each mouse. After 7 days, the mice with similar tumor size (about $150 \mathrm{~mm}^{3}$ ) were randomly allocated to control group $(n=5)$ and treatment group $(n=5)$. The treatment group received intraperitoneal injections of silibinin at the dosage of $100 \mathrm{mg} / \mathrm{kg}$ body weight each day for 12 days, and the control group received vehicle in the same volume. The tumor size was measured by a researcher who was blinded to the group allocation with a slide caliper, and the tumor volume was calculated using the formula: $0.5 \times \mathrm{A} \times \mathrm{B}^{2}$, in which $\mathrm{A}$ is the length of the tumor and $B$ is the width. On the next day of the last treatment, the mice were euthanized by cervical dislocation. After being excised and weighed, the tumors were frozen immediately in liquid nitrogen for western blotting analysis.

\section{Differential centrifugation, gel electrophoresis, and western blotting}

The collected glioma cells by centrifugation and the frozen xenografted glioma tissue were homogenized with a glass Pyrex microhomogenizer (20 strokes) in ice cold lysis buffer (Beyotime Biotech, Nanjing, China). Homogenates were centrifuged at $1000 \times g$ for $10 \mathrm{~min}$ at $4{ }^{\circ} \mathrm{C}$ to obtain the supernatant 1 and the pellet 2 . The supernatant 1 was then centrifuged at $10,000 \times g$ for $10 \mathrm{~min}$ at $4{ }^{\circ} \mathrm{C}$ to obtain supernatant 2 and pellet 2 . The pellet 1 was nuclear fraction, supernatant 1 was cytoplasmic fraction, pellet 2 was mitochondrial fraction, and supernatant 2 was cytoplasmic fraction without mitochondria. The protein content was determined using Bio-Rad protein assay kit. After SDS electrophoresis and transfer to PVDF membranes, the membranes were blocked with 3\% BSA in TBS for $30 \mathrm{~min}$ at room temperature, and then incubated overnight at $4{ }^{\circ} \mathrm{C}$ with primary antibodies. After incubation with horseradish peroxidase-conjugated secondary antibody and washing the blots, immunoreactive proteins were visualized on a chemi-luminescence developer (ChemiScope 5300, Clinx Scicence Instrument Company, Shanghai) and then the density was quantified by using software of Image J. The procedure was performed by a researcher who was blinded to group allocation.

\section{Immunocytochemical staining}

The cells seeded on a culture dish were fixed in ethanol, washed with PBS, and incubated with $1 \%$ Triton X-100 for $10 \mathrm{~min}$. The cells were incubated with $100 \mathrm{nmol} / \mathrm{L}$ Mitotracker red ((Invitrogen company, Eugene, OR)) for $30 \mathrm{~min}$ at $37^{\circ} \mathrm{C}$ before fixation in ethanol. After the nonspecific antibody binding sites were blocked, the cells were incubated with anti-BNIP3 (1:100) or anti-AIF antibody (1:100) followed by incubation in Alexa Fluor 488-conjugated goat anti-rabbit IgG (1:200) for $1 \mathrm{~h}$ and then with Heochst33258. Finally, all the cells were visualized under laser scanning confocal microscope (Olympus FV1000, Tokyo, Japan) by a researcher who was blinded to group allocation.

\section{Measurement of GSH, cysteine, ATP, glucose-6-phophate, and pyruvate}

Intracellular total GSH was measured by using a DTNBGSSH reductase recycling assay kit (Beyotime Biotechnology, Nanjing, China) as described by manufacture. Briefly, the collected cells and frozen glioma tissue were resuspended in protein-removing buffer $\mathrm{S}$ and lysed by repeated cycles of freezing and thawing under liquid nitrogen. The cell lysates were centrifuged at $10,000 \times g$ for $10 \mathrm{~min}$ at $4{ }^{\circ} \mathrm{C}$ to get the supernatant used for assay. GSH content was expressed as a ratio to the absorbance value at $412 \mathrm{~nm}$ of the control cells. 
Intracellular cysteine was measured by using a cysteine assay kit (Nanjing Jiancheng Bioengineering Institute, Nanjing, China) according to the manufacturer protocol. Briefly, the collected cells were added into reagent A, homogenized on ice, and centrifuged at $8000 \times g$ for $4 \mathrm{~min}$ at $4{ }^{\circ} \mathrm{C}$ to obtain the supernatant for assay. After the protein concentration was measured, $20 \mu \mathrm{L}$ sample was incubated with $100 \mu \mathrm{L}$ reagent $B$ and $100 \mu \mathrm{L}$ reagent $C$ for $15 \mathrm{~min}$ at room temperature and read at absorbance $600 \mathrm{~nm}$ in a microplate reader. Finally, the results were expressed as a ratio to the absorbance value of the control cells.

Glucose-6-phosphate, pyruvate, and ATP measurement were all performed according to manufacturer instructions of Biovision company (Milpitas, CA). Briefly, the cells were collected by centrifugation or $10 \mathrm{mg}$ xenograft glioma tissues were lysed in assay buffer by repeated sonification to be prepared to samples for examinations. For glucose-6-phosphate assay, the samples were mixed with respective reaction buffers and readings were taken at $450 \mathrm{~nm}$. For measurement of the concentration of pyruvate and ATP, the samples were mixed with respective reaction buffers and read at absorbance $570 \mathrm{~nm}$ in a microplate reader. All the measurement was performed by researchers who were blinded to group allocation and repeated for five times. Finally, the results were expressed as a ratio to the absorbance value of the control cells.

\section{StubRFP-SensGFP-LC3 assay}

The StubRFP-SensGFP-LC3 assay was performed according to manufacturer's instruction (Genechem, Shanghai China). Briefly, the U87 cells $\left(5 \times 10^{4}\right.$ cells $\left./ \mathrm{mL}\right)$ seeded on a culture dish were incubated with the StubRFP-SensGFP-LC3B lentivirus (MOI $=5 \times 10^{6} \mathrm{TU} /$ $\mathrm{mL})$. New medium was changed after $12 \mathrm{~h}$ of incubation. At transinfection $72 \mathrm{~h}$, the cells were treated with silibinin, and then incubated with lysotracker blue or mitotracker deep red (Invitrogen company, Eugene, OR). Finally, the cells were observed by a researcher who was blinded to group allocation with a confocal laser scanning microscope (Olympus, Tokyo, Japan).

\section{Statistical analysis}

All data represent at least four independent experiments and are expressed as mean \pm SD. Statistical comparisons were made using one-way ANOVA. $P$ values of less than 0.05 were considered to represent statistical significance.

\section{Results}

\section{Autophagy contributed to silibinin-induced glioma cell death}

To evaluate the toxic effect of silibinin on glioma cells, we used MTT assay to assess cellular viability. As shown in Fig. 1b, treatment with silibinin at indicated concentrations for $24 \mathrm{~h}$ resulted in obvious decreases in the cellular viabilities of U87, U251 and SHG-44 and C6 glioma cells, and the reduction of cellular viability became more apparent with the increase of silibinin concentration. This indicated that silibinin inhibited glioma cell viability in a dosage-dependent manner. Then, we calculated the IC50 values of silibinin at $24 \mathrm{~h}$ and found that they were $272.1 \mu \mathrm{mol} / \mathrm{L}$ in U87 cells, $261.6 \mu \mathrm{mol} / \mathrm{L}$ in U251 cells, $246.1 \mu \mathrm{mol} / \mathrm{L}$ in SHG-44 cells, and $162.5 \mu \mathrm{mol} / \mathrm{L}$ in $\mathrm{C} 6$ cells. Thus, $200 \mu \mathrm{mol} / \mathrm{L}$ was used in the subsequent studies.

To address the mechanism accounting for the inhibitory effect of silibinin on glioma cells, we investigated whether silibinin induced activation of lethal autophagy. As revealed by western blotting analysis, silibinin triggered time-dependent increases of autophagy marker proteins ATG5 and LC3-II, and corresponding reduction of autophagy substrate p62 (SQSTM1) (Fig. 1c and Fig. S1A-C). Then, the U87 cells transinfected with stubRFP-sensGFP-LC3 lentiviruses were used to evaluate whether autophagy flux was activated by silibinin in glioma cells. As revealed by confocal microscopy combined with lysosome probe lysotracker blue staining, many red pucta and green punta were found to form in the cytoplasm of the cells treated with silibinin at $200 \mu \mathrm{mol} / \mathrm{L}$ for $10 \mathrm{~h}$, when compared with control cells (Fig. 1d). Although some red puncta were colocalized with green puncta to display yellow color, the other red puncta were colocalized with blue puncta to present violet color (Fig. 1d). Because green fluorescence is prone to be quenched in the acid environment of lysosome lumen, the yellow puncta were autophagosomes and the violet puncta were autolysosomes. Therefore, these results indicated that silibinin activated autophagy.

To assay the role of autophagy in silibinin-induced glioma cell death, U87 and U251 cells were treated for $1 \mathrm{~h}$ with 3MA that inhibits autophagy at initiation stage or bafilomycin A1 that disturbs autophagosome to fuse with lysosome, and then incubated with silibinin at $200 \mu \mathrm{mol} / \mathrm{L}$ for $24 \mathrm{~h}$. Western blotting analysis showed that silibinininduced upregulation of LC3-II and downregulation of p62 (SQSTM1) were both obviously inhibited by pretreatment with $5 \mathrm{mmol} / \mathrm{L} 3 \mathrm{MA}$. Despite the reduction of p62 (SQSTM1) induced by silibinin was markedly suppressed in the cells pretreated with $1.5 \mu \mathrm{mol} / \mathrm{L}$ bafilomycin A1, the protein level of LC3-II was further improved (Fig. 1e, Fig. S1D and E). Then, LDH release assay demonstrated that silibinin-induced death in glioma cells was significantly abrogated in the presence of 3MA or bafilomycin A1 (Fig. 1f). To further verify the role of autophagy in silibinin-induced glioma cell death, we knocked down ATG5 with SiRNA and examined its effect on glioma cell death. It was found that knockdown of ATG5 not only prevented silibinin-induced upregulation 
of LC3-II and downregulation of p62 (SQSTM1) (Fig. 1g, Fig. S1F-H), but also inhibited glioma cell death (Fig. 1h). Therefore, these results indicated that silibinin induced autophagic death in glioma cells.

Furthermore, we used SHG-44 cells to investigate whether silibinin induced necroptosis and ferroptosis in glioma cells. As shown by LDH assay, $1 \mathrm{~h}$ pretreatment with necroptosis specific inhibitor Nec-1 $(100 \mu \mathrm{mol} / \mathrm{L})$, GSK-872 $(20 \mu \mathrm{mol} / \mathrm{L})$, and NSA $(10 \mu \mathrm{mol} / \mathrm{L})$ did not prevent silibinin-induced glioma cell death (Fig. S1I). Additionally, pretreatment with ferroptosis inhibitor deferoxamine $(500 \mu \mathrm{mol} / \mathrm{L})$ and Fer-1 $(20 \mu \mathrm{mol} / \mathrm{L})$ did not inhibit the glioma cell death induced by silibinin as well (Fig. S1J). Thus, these results indicated that neither necroptosis nor ferroptosis was induced in glioma cells by silibinin.

\section{Autophagy contributed to silibinin-induced mitochondria damage}

To address whether silibinin induces damage in mitochondrion, we measured mitochondrial membrane potential by using JC- 1 and mitochondrial superoxide by using Mitosox red. JC-1is a probe emitting red fluorescence when accumulates in healthy mitochondria but green fluorescence when is released from damaged mitochondria into cytoplasm. As revealed by fluorescence microscopy, the red fluorescence exhibited by JC-1 decreased drastically in the cells treated with silibinin at $200 \mu \mathrm{mol} / \mathrm{L}$ for $12 \mathrm{~h}$, when compared with control cells (Fig. 2a). This was also demonstrated by the results acquired by flow cytometry analysis combined with JC-1 staining, which showed that silibinin caused timedependent reduction in red fluorescence (Fig. 2b, Fig. S2A). These indicated that silibinin induced mitochondria depolarization in a time-dependent manner. Then, we found that the red fluorescence exhibited by Mitosox red which is a specific probe for mitochondrial superoxide was much brighter in the cells treated with silibinin at $200 \mu \mathrm{mol} / \mathrm{L}$ for $24 \mathrm{~h}$ than that in control cells (Fig. 2c). Statistical analysis demonstrated as well that the red fluorescence intensity was improved with the extension of silibinin treatment (Fig. 2d). This indicated that silibinin induced time-dependent accumulation of mitochondrial superoxide. Therefore, these data suggested that silibinin treatment resulted in mitochondrial damage.

Considering that AIF translocation from depolarized mitochondria to nuclei could lead to cell death, we thus isolated mitochondrial and nuclear fractions and examined silibinin-induced changes in distribution of AIF. As revealed by western blotting, when compared with control cells, AIF was decreased in mitochondria fractions, whereas increased in nuclear fractions by silibinin in a time-dependent manner (Fig. 2e, Fig. S2B-D). Confocal microscopy showed as well that AIF accumulated more apparently in the nuclei of the cells incubated with silibinin for $24 \mathrm{~h}$ than that in control ones (Fig. 2f). To clarify the role of AIF in silibinin-induced glioma cell death, we introduce SiRNA to knock down AIF and examined its effect on glioma cell death by using LDH release assay. It was found that silibinin-induced upregulation of AIF in nuclear fractions was mitigated as a consequence of the overall reduction of AIF expression in the cells transfected with AIF SiRNA when compared with the cells transfected with scrambled SiRNA (Fig. 2g, Fig. S2E-G). Moreover, silibinin-induced glioma cell death was also suppressed significantly when AIF was knocked down with SiRNA (Fig. 2h). Therefore, these indicated that silibinin triggered AIF-dependent death in glioma cells.

Furthermore, we found that inhibition of autophagy with 3MA or bafilomycin A1 obviously abrogated silibinin-induced accumulation of mitochondrial superoxide and AIF translocation from mitochondria to nuclei (Fig. 2i and j, Fig. S2H-J). Similar results could be found when ATG5 was knocked down with SiRNA (Fig. 2k and l, Fig. S2K-M). Therefore, these results suggested that autophagy contributed to silibinin-induced mitochondria damage and nuclear translocation of AIF.

\section{Autophagy reinforced silibinin-induced BNIP3 accumulation on mitochondria}

Given that BNIP3 (Bcl-2/adenovirus E1B 19-kDainteracting protein 3 ) is protein targeting mitochondria and could induce mitochondrial damage and nuclear translocation of $\mathrm{AIF}^{6}$, we thus isolated mitochondrial fractions from U87, U251, SHG-44, and C6 cells and examined silibinin-induced changes in BNIP3. As shown by western blotting, silibinin not only induced BNIP3 overexpression, but also improved BNIP3 levels in mitochondrial fractions in a time-dependent manner (Fig. 3a, Fig. S3A and B). Confocal microscopy also confirmed that BNIP3 accumulated more obviously on mitochondria in the cells treated with silibinin for $24 \mathrm{~h}$ than that in control cells (Fig. 3b). These indicated that silibinin promoted BNIP3 accumulation on mitochondria, as well as upregulated its expression in glioma cells. To elucidate the role of BNIP3 in silibinin-produced toxicity in glioma cells, we used SiRNA to knock down BNIP3 and examined its effect on glioma cell death and mitochondrial damage. As revealed by western blotting, silibinin-induced BNIP3 overexpression and its accumulation on mitochondria were both inhibited in the cells transfected with BNIP3 SiRNA, when compared with the cells transfected with scrambled SiRNA (Fig. 3c, Fig. S3C and D). Moreover, LDH release assay proved that knockdown of BNIP3 with SiRNA significantly prevented the glioma cell death induced by silibinin (Fig. 3d). Furthermore, silibinininduced accumulation of mitochondrial superoxide, mitochondria depolarization, and AIF translocation from mitochondria to nuclei were all inhibited when BNIP3 was knocked down with SiRNA (Fig. 3e-g, Fig. S3E-G). 


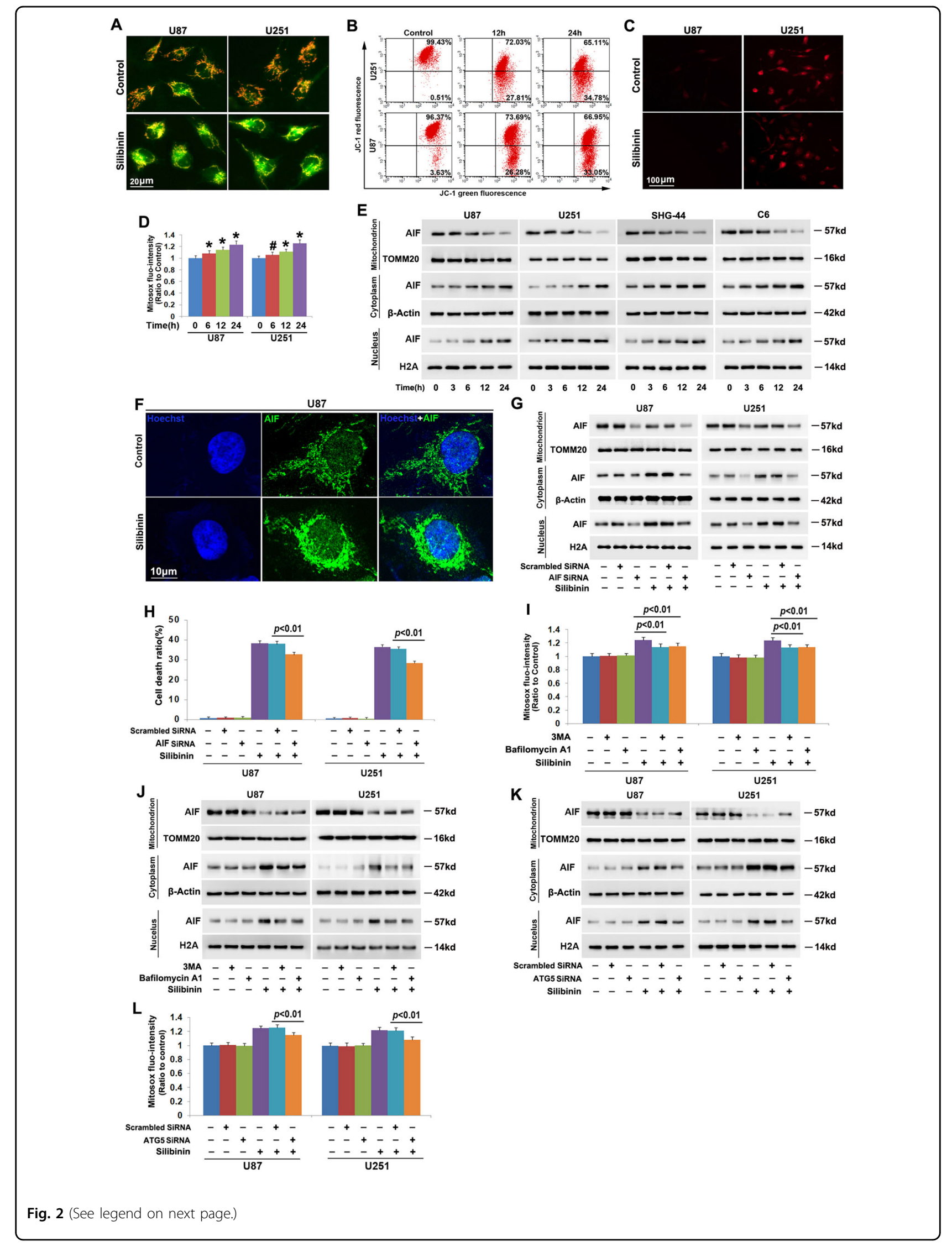


(see figure on previous page)

Fig. 2 Autophagy contributed to silibinin-induced mitochondria damage. a Representative images of the cells stained with JC-1 under fluorescence microscope showed that silibinin treatment resulted in obvious reduction of red fluorescence in U87 and U251 cells. b Flow cytometry analysis combined with JC-1 staining confirmed that silibinin induced time-dependent dissipation of mitochondria membrane potentials. c Representative images of the cells stained with Mitosox red under fluorescence microscope showed that the red fluorescence exhibited by Mitosox red was apparently stronger in silibinin-treated cells than that in control group. $\mathbf{d}$ Statistical analysis of the red fluorescence intensity exhibited by Mitosox red proved that silibinin triggered mitochondrial accumulation of superoxide in a time-dependent manner. e Western blotting analysis revealed that silibinin induced AIF translocation from mitochondria to nuclei in a time-dependent manner. $\mathbf{f}$ Representative images acquired by confocal microscopy combined with immunochemical staining showed that silibinin induced accumulation of AIF in the nucleus of U87 cell. $\mathbf{g}$ Silibinin-induced accumulation of AIF in nuclear fraction was decreased in the cells transfected with AIF SiRNA. $\mathbf{h}$ LDH release assay proved that knockdown of AIF prevented silibinin-induced glioma cell death. i Statistical analysis of the red fluorescence intensity exhibited by Mitosox red showed that silibinin-induced mitochondrial accumulation of superoxide was significantly inhibited in the presence of 3MA or bafilomycin A1. j Western blotting proved that knockdown of ATG5 with SiRNA prevented silibinin-induced AIF translocation from mitochondria to nuclei. $\mathbf{k}$ Western blotting revealed that silibinin-induced nuclear translocation of AIF was suppressed when ATG5 was knocked down with SiRNA. I Knockdown of ATG5 with SiRNA abrogated the improvement of mitochondrial superoxide induced by silibinin.

Thus, these indicated that BNIP3 contributed to silibinininduced glioma cell death via causing mitochondrial damage and nuclear translocation of AIF.

Notably, we found that silibinin-induced overexpression of BNIP3 and BNIP3 upregulation in mitochondrial fractions were both inhibited in the cells pretreated with 3MA or bafilomycin A1 (Fig. 3h, Fig. S3H and I). This indicated that autophagy promoted silibinin-induced BNIP3 overexpression and its accumulation on mitochondria. This might be a factor accounting for the promotion effect of autophagy on silibinin-induced mitochondria.

\section{Autophagy augmented silibinin-induced accumulation of $\mathrm{H} 2 \mathrm{O} 2$}

To address the mechanism accounting for the regulatory effect of silibinin on BNIP3, we examined silibinin-induced changes in HIF- $1 \alpha$ and hydrogen peroxide given that HIF- $1 \alpha$ is a transcription factor of BNIP3 and BNIP3 could also be upregulated under the condition of oxidative stress ${ }^{6}$. Thus, we isolated nuclear fractions by using differential centrifugation and examined the protein level of HIF- $1 \alpha$ in the absence and presence of silibinin by western blotting. When compared that in control cells, nuclear level of HIF-1 $\alpha$ was downregulated by silibinin in a time-dependent manner (Fig. 4a, Fig. S4A). However, intracellular hydrogen peroxide was markedly improved time-dependently by silibinin, which was accompanied with GSH depletion at each indicated time point (Fig. 4b). Considering that NADPH oxidase (Nox) plays a role in promoting the generation of hydrogen peroxide, the cells were pretreated with Nox inhibitor GKT137831 at $500 \mu \mathrm{mol} / \mathrm{L}$ for $1 \mathrm{~h}$ and then incubated with silibinin for $24 \mathrm{~h}$. It was found that GKT13831 not only inhibited silibinin-induced improvement of hydrogen peroxide, but also prevented glioma cell death (Fig. S4B and C). This indicated that Nox might be involved in regulation silibinin-induced overproduction of hydrogen peroxide. Furthermore, we found that silibinin treatment also resulted in time-dependent upregulation of glutathione peroxidase 4 (GPX4) (Fig. 4a, Fig. S4D). Given that GSH could be used by GPX4 to clear hydrogen peroxide ${ }^{17}$, the glioma cells were supplemented with exogenous GSH to verify the role of hydrogen peroxide in silibinin-induced changes in BNIP3. It was found that pretreatment with GSH at $10 \mathrm{mmol} / \mathrm{L}$ for $1 \mathrm{~h}$ obviously inhibited the improvement of hydrogen peroxide induced by silibinin at indicated dosages (Fig. 4d). Moreover, LDH release assay demonstrated as well that silibinin-induced glioma cell death was abrogated significantly by supplement of GSH (Fig. 4e). Then, it was revealed by western blotting that silibinin-induced overexpression of BNIP3 in cytoplasmic and mitochondrial fractions were both inhibited in the presence of GSH (Fig. 4f, Fig. S4E and F). To further confirm the role of hydrogen peroxide in promoting BNIP3 expression and accumulation on mitochondria, we treated U87 and U251 cells with exogenous hydrogen peroxide at $300 \mu \mathrm{mol} / \mathrm{L}$ for indicated time and analyzed the distribution of BNIP3 by western blotting. It was found that hydrogen peroxide not only upregulated BNIP3 expression, but also improved BNIP3 levels in mitochondrial fractions in a time-dependent manner (Fig. 4g, Fig. S4G and F). These results indicated that GSH depletion contributed to silibinin-induced improvement of hydrogen peroxide, which resulted in BNIP3 upregulation and accumulation on mitochondria.

Additionally, we found that pretreatment with 3MA or bafilomycin A1 not only reversed silibinin-induced GSH depletion, but also prevented accumulation of hydrogen peroxide (Fig. $4 \mathrm{~h}$ and i). Therefore, these results suggested that autophagy contributed to silibinin-induced changes in BNIP3 via causing GSH depletion-dependent accumulation of hydrogen peroxide.

\section{Autophagy promoted silibinin-induced phosphorylation of p53}

To elucidate why silibinin treatment leads to GSH depletion, we examined silibinin-induced changes in cysteine because it is a material used for GSH synthesis ${ }^{21}$. 


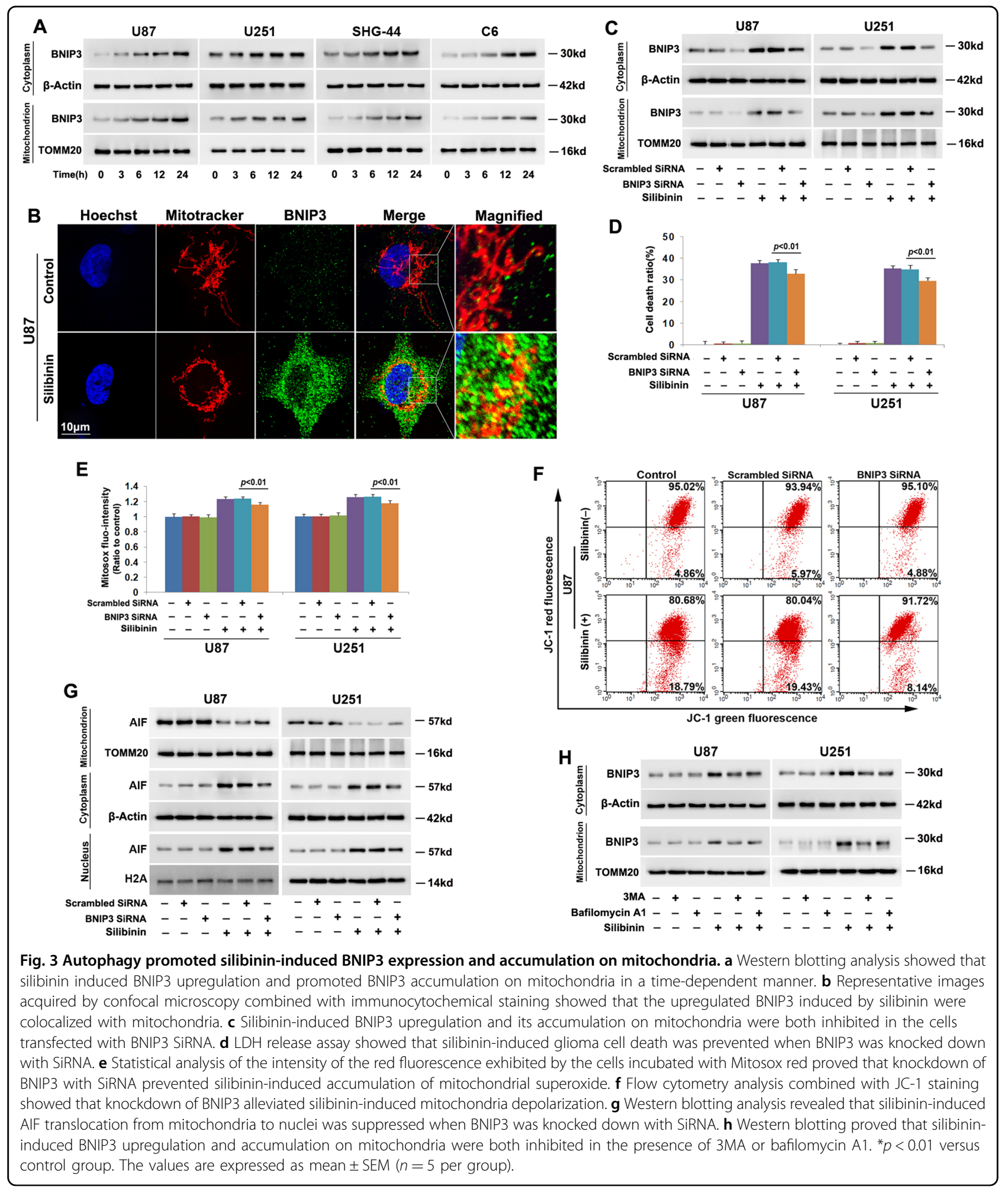

As shown in Fig. 5a, intracellular cysteine was decreased by silibinin in a time-dependent manner, when compared with that in control cells. Considering that cysteine is transformed from cystine whose transportation into cells depends on cystine/glutamate antiporter ${ }^{21}$, we used western blotting to analyze silibinin-induced changes in the protein level of $x C T$ (SLC7A11) and phosphorylation of p53. xCT is a specific light-chain subunit of cystine/glutamate 


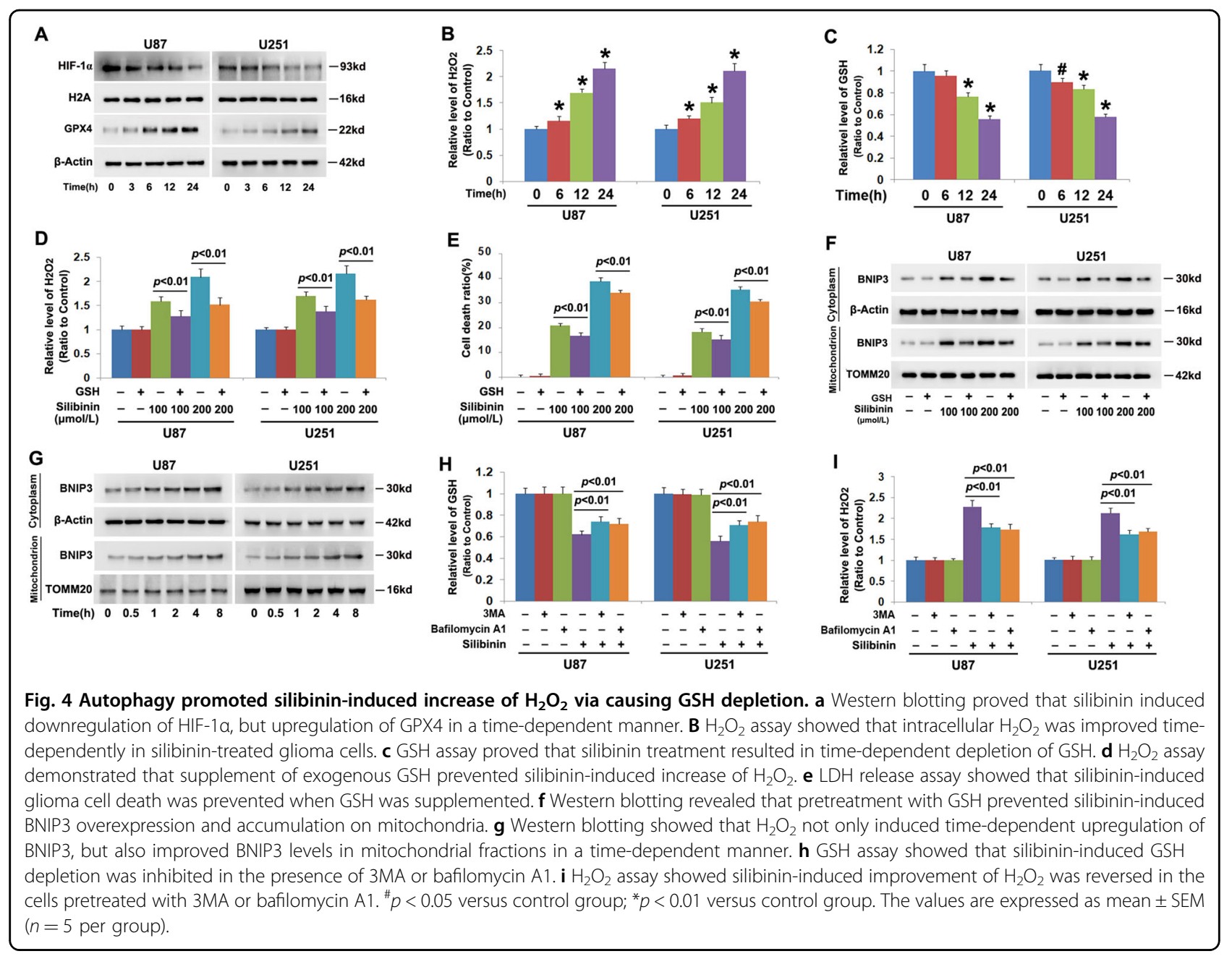

antiporter and its expression is negatively regulated by phosphorylated $\mathrm{p} 53^{21}$. We found that silibinin downregulated $\mathrm{xCT}$, but upregulated $\mathrm{p} 53$ and phospho-p53 in a time-dependent manner (Fig. 5b, Fig. S5A-C). These indicated that silibinin activated $\mathrm{p} 53$, which resulted in downregulation of $\mathrm{xCT}$ and depletion of cysteine.

In contrast, the downregulation of $\mathrm{xCT}$ and the phosphorylation of p53 induced by silibinin were both inhibited apparently when the cells were pretreated with 3MA or bafilomycin A1 (Fig. 5c, Fig. S5D-F). Moreover, silibinininduced depletion of cysteine was correspondingly prevented in the presence of 3MA or bafilomycin A1 (Fig. 5d). Similar results could be found when ATG5 was knocked down with SiRNA (Fig. 5e and f). Thus, these data indicated that autophagy contributed to silibinin-induced depletion of cysteine via promotion of p53 activation.

\section{Silibinin suppressed glycolysis in glioma cells}

To elucidate the mechanism accounting for silibinininduced autophagy activation, we investigated whether silibinin induces glycolysis dysfunction considering that glycolysis is the primary energy-generating pathway in cancer cells and energy failure could lead to autophagy activation ${ }^{4}$. It was found that silibinin triggered timedependent depletion of ATP (Fig. 6a). Moreover, glycose6-phosphate, which is the first product of glycolysis generated by hexokinase II (HK II) and pyruvate that is the final product of glycolysis produced by pyruvate kinase 2 (PKM2) were both depleted by silibinin in a timedependent manner (Fig. $6 \mathrm{~b}$ and c). These indicated that silibinin suppressed glycolysis in glioma cells. Then, western blotting was used to analyze silibinin-induced changes in HK II, 6-phosphofructokinase (PFKP), and PKM2, which are known to regulate the three ratelimiting steps of glycolysis. We found that the protein levels of HK II, PFKP, and PKM2 were all downregulated time-dependently by silibinin in U87, U251, SHG-44, and C6 glioma cells (Fig. 6d, Fig. S6A-C). This indicated that silibinin suppressed glycolysis via downregulation of $\mathrm{HK}$ II, PFKP, and PKM2.

To test the role of hydrogen peroxide in silibinininduced glycolysis dysfunction, U87 and U251 cells were 


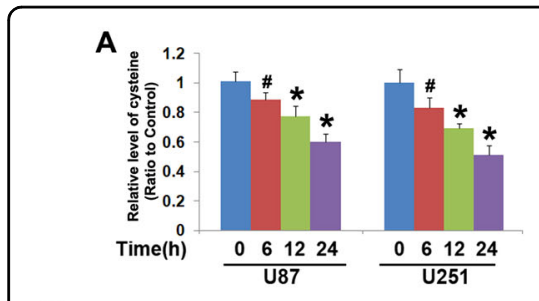

D
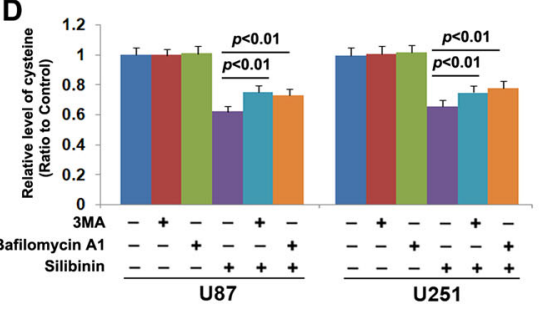

B

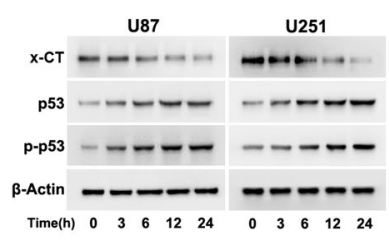

E

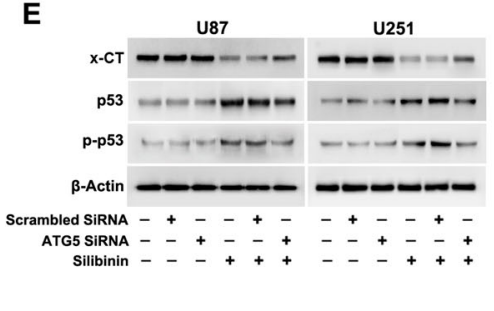

Fig. 5 Autophagy promoted silibinin-induced cystine depletion and p53 phosphorylation. a Cysteine assay showed that silibinin induced time-dependent depletion of cysteine. b, c Western blotting analysis proved that silibinin downregulated xCT, but upregulated p53 and phosphop53 in a time-dependent manner. These were all prevented in the presence of 3MA or bafilomycin A1. $\mathbf{d}$ Cysteine assay demonstrated that silibinininduced depletion of cysteine was prevented in the presence of 3MA or bafilomycin A1. e Western blotting demonstrated that knockdown of ATG5 with SiRNA prevented silibinin-induced downregulation of CT and upregulation of p53 and phospho-p53. $\mathbf{f}$ Cysteine assay proved that silibinininduced depletion of cysteine was inhibited when ATG5 was knocked down with SiRNA. ${ }^{\#} p<0.05$ versus control group; ${ }^{*} p<0.01$ versus control group. The values are expressed as mean \pm SEM ( $n=5$ per group).

treated with antioxidant GSH at $10 \mathrm{mmol} / \mathrm{L}$ for $1 \mathrm{~h}$ and then incubated with silibinin at $200 \mu \mathrm{mol} / \mathrm{L}$ for $24 \mathrm{~h}$. It was found that silibinin-induced reduction of ATP, glucose-6-phosphate, and pyruvate were all inhibited by supplement of GSH (Fig. 6e-g). Western blotting analysis revealed that pretreatment with GSH obviously abrogated the downregulation of HK II, PFKP, and PKM2 induced by silibinin at indicated dosages (Fig. 6h, Fig. S6D-F). Correspondingly, silibinin-induced increases of autophagy marker proteins ATG5 and LC3-II and decrease of autophagy substrate p62 (SQSTM1) were all inhibited in the presence of GSH (Fig. 6h, Fig. S6G-I). Thus, these data not only indicated that hydrogen peroxide reinforced the inhibitory effect of silibinin on glycolysis, but also indirectly suggested that glycolysis dysfunction led to silibinin-induced autophagy activation.

\section{Silibinin inhibited glioma cell growth in vivo}

To verify the treatment effect of silibinin on glioma cells in vivo, C6 cells were xenografted subcutaneously into the flank of nude mice. After being treated with silibinin at the dosage of $100 \mathrm{mg} / \mathrm{kg}$ each day for consecutive 12 days, the volumes of the xenografted tumors were found to decrease markedly when compared with those in control group (Fig. 7a). This was also confirmed by statistical analysis of tumor volumes (Fig. 7b). Then, the tumors removed from the sacrificed mice were homogenized and silibinin-induced changes in autophagy marker proteins were analyzed by western blotting. When compared with the control group, silibinin triggered obviously upregulation of ATG5 and LC3-II, but reduction of p62
(SQSTM1) (Fig. 7c, Fig. S7A). This indicated that silibinin induced autophagy activation in glioma cells in vivo.

Moreover, it was found that hydrogen peroxide was at higher level, whereas GSH and cysteine were both at lower levels in silibinin-treated group than control group (Fig. 7d-f). Consistently, western blotting analysis revealed that $\mathrm{xCT}$ was markedly downregulated, but $\mathrm{p} 53$ and phospho-p53 were both obviously upregulated by silibinin (Fig. 7g, Fig. S7B). This indicated silibinininduced accumulation of hydrogen peroxide, depletion of GSH and cysteine, downregulation of $\mathrm{xCT}$ and activation of p53 in glioma cells in vivo.

Furthermore, we found that silibinin not only upregulated the expression of BNIP3, but also improved BNIP3 levels in mitochondrial fractions (Fig. 7h, Fig. S7C). Although AIF was decreased by silibinin in mitochondrial fractions, it was increased apparently in nuclear fractions (Fig. 7i, Fig. S7D). This indicated that BNIP3 might play a role in regulation of silibinin-induced nuclear translocation of AIF in glioma cells in vivo. Additionally, it was found that silibinin treatment resulted in depletion of ATP, glucose-6-phosphate, and pyruvate (Fig. 7j-l) and downregulation of HK II, PFKP, and PKM2 (Fig. $7 \mathrm{~m}$, Fig. S7E). This indicated that silibinin triggered energy failure via suppression of glycolysis in glioma cells in vivo.

\section{Discussion}

In summary, we found in this study that silibinin induced AIF translocation from mitochondria to nuclei in glioma cells in vitro and in vivo, which was accompanied with autophagy activation. In vitro studies revealed that 


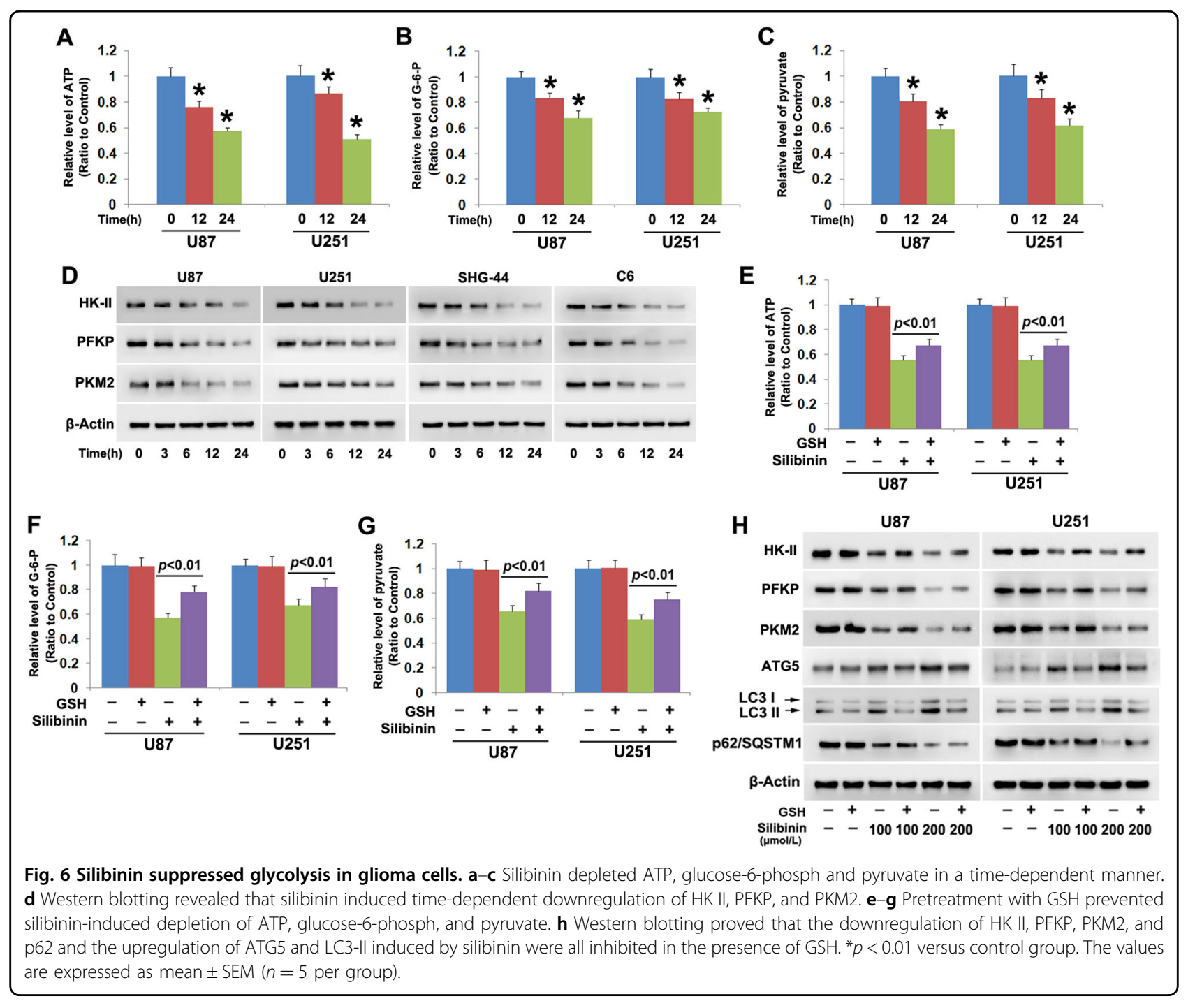

blocking autophagy with 3MA1, bafilomycin A1 or by knocking down ATG5 with SiRNA inhibited silibinininduced mitochondrial accumulation of superoxide, AIF translocation from mitochondria to nuclei and glioma cell death. Mechanistically, silibinin activated autophagy through depleting ATP by suppressing glycolysis. Then, autophagy improved $\mathrm{H}_{2} \mathrm{O}_{2}$ via promoting p53-mediated depletion of GSH and cysteine and downregulation of xCT. The increased $\mathrm{H}_{2} \mathrm{O}_{2}$ not only contributed to silibinin-induced BNIP3 upregulation and translocation to mitochondria, but also exacerbated glycolysis dysfunction. Knockdown of BNIP3 with SiRNA inhibited silibinin-induced mitochondrial depolarization, accumulation of mitochondrial superoxide, and AIF translocation from mitochondria to nuclei, as well as prevented glioma cell death. Collectively, autophagy contributed to silibinin-induced glioma cell death via causing BNIP3- mediated mitochondria damage and nuclear translocation of AIF (Fig. 8).

Overactivation of autophagy has been established to be an effective way to eradicate cancer cells ${ }^{7,8,22}$, and silibinin is confirmed to be a potent lethal autophagy inducer for various types of cancer cells such as human breast cancer cells, fibrosarcoma cells, and renal carcinoma cells ${ }^{7,8,23}$. Different from previous report showing that silibinin triggered protective autophagy in glioma cells ${ }^{24}$, we found in this study that blocking autophagy with 3MA, bafilomycin A1 or by knocking down ATG5 with SiRNA significantly rescued silibinin-induced glioma cell death. This verified that autophagy contributed to silibinininduced death in glioma cells. Moreover, different types of programmed cell death could be induced by the same chemical or under the same pathological condition. Previous reports have shown that silibinin treatment could 


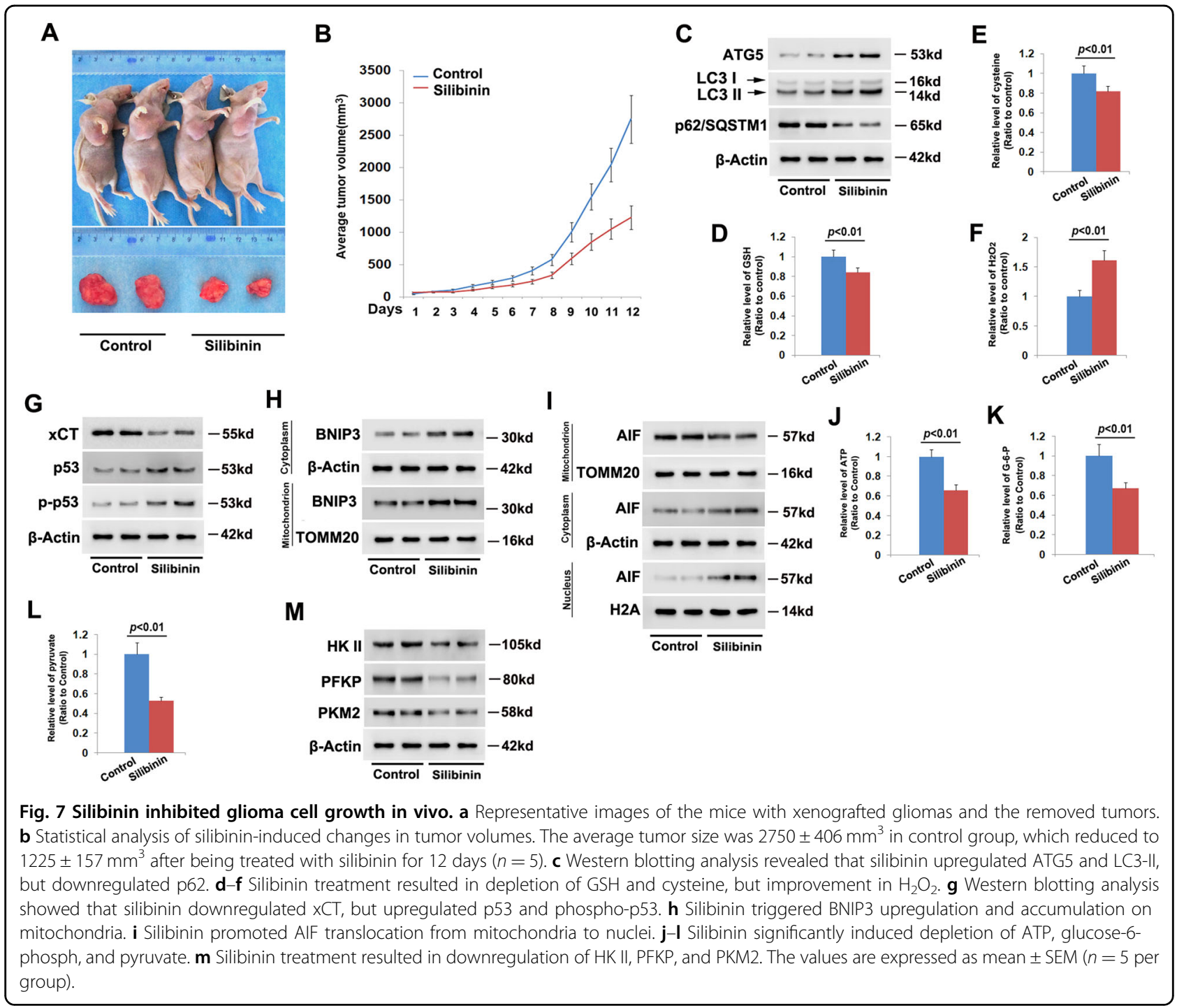

also trigger apoptosis in glioma cells ${ }^{15}$. Additionally, accumulating evidences have demonstrated that autophagic death could occur simultaneously with other types of programmed cell death, such as ferroptosis and necroptosis ${ }^{25,26}$. Thus, induction of autophagic death is a pathway accounting for the toxic effect of silibinin on glioma cells.

Energy failure is a key factor initiating autophagy via activation of AMPK/mTOR pathway ${ }^{4}$. Glycolysis regulated by three rate-limiting enzymes HK II, PFKP, and PKM2 is usually used by cancer cells to acquire $\mathrm{ATP}^{20}$. Suppression of glycolysis by pharmacological inhibition of HK II and genetic knockdown of PKM2 were respectively reported to causing cancer cell death via excessive activation of autophagy $y^{27,28}$. In this study, we found that treatment with silibinin not only obviously reduced ATP level, but also depleted glucose-6-phosphate and pyruvate and downregulated the protein levels of HK II, PFKP, and
PKM2. This suggested that silibinin induced energy failure in glioma cells via suppressing glycolysis, which was also supported by the study showing that silibinin suppressed glycolytic activity in pancreatic cancer cells ${ }^{29}$. Although we did not further investigate whether AMPK/ mTOR pathway was activated by silibinin, AMPK/mTOR activation was reported to account for silibinin-triggered autophagic death in renal carcinoma cells ${ }^{23}$. Thus, we thought that silibinin induced lethal autophagy in glioma cells via suppression of glycolysis.

Despite it was generally accepted that the protection of autophagy against cell death is associated with inhibition of oxidative stress by clearing damaged mitochondria ${ }^{5}$, several studies have shown that autophagy plays a role in promotion of oxidative stress. Autophagy was reported to improve intracellular ROS levels via selective degradation of catalase, which is an enzyme responsible for clearing hydrogen peroxide ${ }^{30}$. Moreover, it was also found that HK 


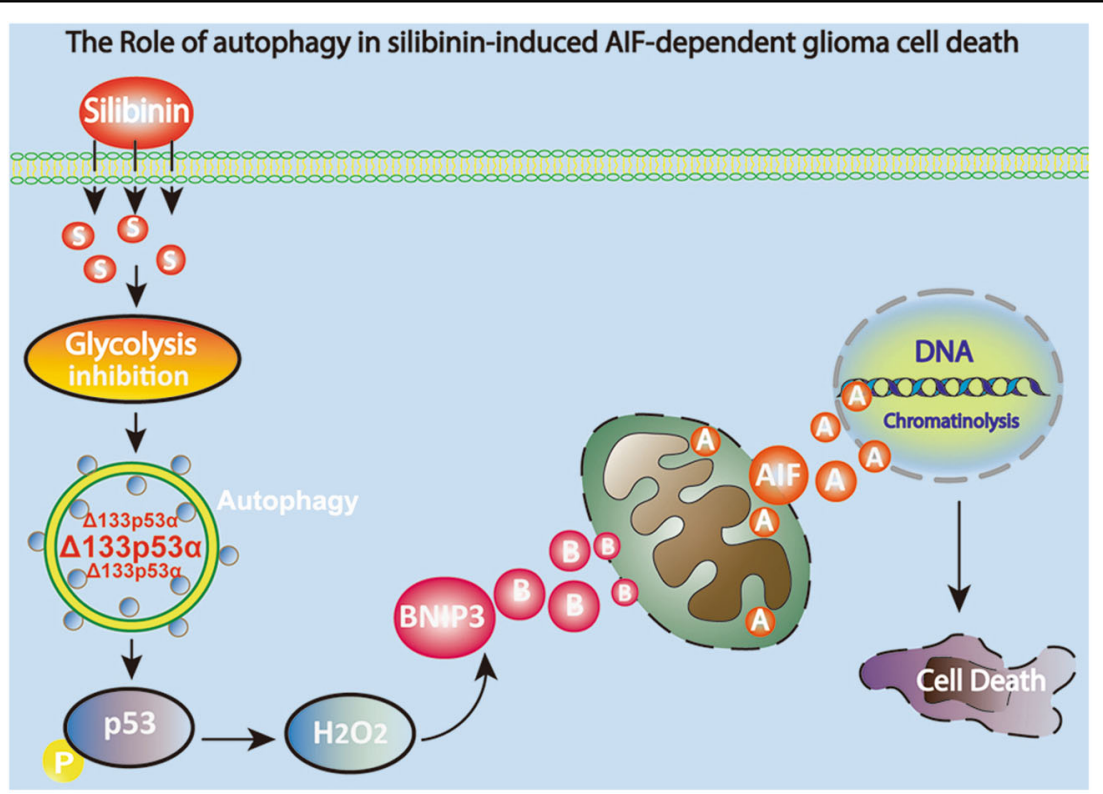

Fig. 8 Schematic diagram for the role of autophagy in silibinin-induced mitochondrial damage and AlF-dependent glioma cell death. Silibinin inhibits glycolysis in glioma cells, which results in autophagy activation. The activated autophagy selectively degrades $\Delta 133 p 53 a$ (an inhibitoryp 53 isoform) to improve p53 phosphorylation. Then, the phosphorylated p53 reinforces generation of hydrogen peroxide, which further promotes the expression of BNIP3. BNIP3 damages mitochondria and causes AIF translocate from mitochondrion into nucleus. Finally, AIF leads to cell death via causing chromatinolysis.

II which accounts for generating glucose-6-phosphate during the process of glycolysis is also a selective substrate of autophagy $^{31}$. Notably, knockdown of HK II with SiRNA was confirmed to cause accumulation of intracellular $\operatorname{ROS}^{32}$. Thus, these studies revealed that autophagy also plays an important role in improving intracellular ROS levels. Accumulating evidences showed as well that oxidative stress was responsible for silibinin-induced death in various types of cancer cells ${ }^{8,12,33}$. Recent studies proved that activation of p53 is a primary pathway via which silibinin improved intracellular ROS levels. Activated p53 promoted silibinin-induced oxidative stress mainly through two pathways, one is triggering excessive generation of ROS in mitochondria by activation of JNK, and the other is causing depletion of antioxidant $\mathrm{GSH}^{34,35}$. Of note, activated p53 was reported to deplete GSH via negative regulation of cysteine level and $\mathrm{xCT}$ expres$\operatorname{sion}^{21}$. In this study, we found that blocking autophagy with 3MA or bafilomycin A1 not only inhibited silibinininduced phosphorylation of p53, but also prevented silibinin-triggered downregulation of $\mathrm{xCT}$, depletion of cysteine and GSH and improvement of hydrogen peroxide. Although we did not investigate in this study the role of autophagy in silibinin-induced activation of p53, it was reported that autophagy promoted p53 activation via selectively degradation of $\Delta 133 \mathrm{p} 53 \alpha$ (an inhibitory p 53 isoform that can inhibit full-length $\mathrm{p} 53)^{36}$. Therefore, our data suggested that autophagy contributed to silibinin- induced oxidative stress in glioma cells via promotion of p53 activation. Additionally, it was also reported that glycolysis was inhibited significantly when glioma cells were treated with hydrogen peroxide ${ }^{20}$. In this study, we found that the inhibitory effect of silibinin on glycolysis was apparently reversed when hydrogen peroxide was suppressed by supplement of GSH. Therefore, silibinin also induced a positive feedback between glycolysis dysfunction and autophagy, which resulted in excessive activation of autophagy.

Autophagy was reported to account for the glioma cell death induced by ceremide or compound AT101 via excessively removing mitochondria ${ }^{10,11}$, whereas accumulating evidences have shown that only damaged mitochondria, which are separated from mitochondrial network could be recognized, encapsulated and then degraded via autophagy pathway ${ }^{37}$. Thus, mitochondria damage is prior to its removal by autophagy. It has been demonstrated that apoptosis, necroptosis, and parthanatos contributed to cell death via causing mitochondrial damage and nuclear translocation of $\mathrm{AIF}^{38-40}$, but it remains elusive whether autophagy plays a role in impairing mitochondria and promoting AIF translocation from mitochondria to nuclei. In this study, we found that blocking autophagy with 3MA, bafilomycin A1 or by knocking down ATG5 with SiRNA not only inhibited silibinin-induced glioma cell death, but also prevented mitochondrial accumulation of superoxide and nuclear 
translocation of AIF. As a protein located within the space between mitochondria outer membrane and inner membrane, AIF is only released from damaged mitochondria, which had depleted membrane potential ${ }^{41,42}$. Within nucleus, AIF acts as a nuclease after cooperating with cyclophilin A to form a DNA-degrading complex at the location of DNA double strand breaks, which eventually leads to irreversible chromatinolysis and cell death ${ }^{41,42}$. Consistent with previous report showing that silibinin induced glioma cell death via promotion of AIF translocation from mitochondria to nuclei ${ }^{15}$, our data in this study proved that knockdown of AIF with SiRNA not only prevented silibinin-induced its accumulation in nuclei, but also inhibited glioma cell death. Thus, autophagy contributes to silibinin-induced glioma cell death via causing mitochondria damage and AIF translocation form mitochondria to nuclei. Previously, intracellular ROS were found to play a crucial role in promotion of mitochondria damage and AIF translocation from mitochondria to nuclei. It was reported that treatment with hydrogen peroxide obviously triggered mitochondrial depolarization and nuclear translocation of AIF in glioma cells ${ }^{40}$. Therefore, these studies suggested that improvement of intracellular hydrogen peroxide is a pathway via which autophagy promoted silibinin-induced mitochondrial damage.

As a protein targeting mitochondria, BNIP3 is excessively expressed in human gliomas ${ }^{43}$. BNIP3 plays dual roles in regulation of cell demise. Suppression of BNIP3 by upregulation of miR-145 was reported to induce apoptosis in glioma cells ${ }^{43}$. However, BNIP3 upregulation could also lead to cell death. It was reported that overexpressed BNIP3 contributed to baicalein-induced apoptosis in osteosarcoma cells, hydrogen peroxide-induced autophagic death in glioma cells, and doxorubicininduced necrosis in cardiac myocytes ${ }^{44,45}$. As well as Bax, Bim and Noxa which induce mitochondrial dysfunction upon accumulation on mitochondria ${ }^{46-48}$, BNIP3 was confirmed to trigger MPTP opening and loss of mitochondrial membrane potentials when its Cterminal transmembrane domain is inserted into mitochondrial outer membrane ${ }^{10}$. Jiang et al. proved that BNIP3 was involved in regulation of silibinin-induced reduction of mitochondrial membrane potentials in human breast cancer cells ${ }^{7}$. Consistently, we found in this study that silibinin not only upregulated the protein level of BNIP3, but also promoted BNIP3 translocate to mitochondria. In contrast, knockdown of BNIP3 with SiRNA obviously inhibited silibinin-induced mitochondrial depolarization, accumulation of mitochondrial superoxide and nuclear translocation of AIF, as well as rescued glioma cell death. Thus, BNIP3 contributed to silibinin-induced glioma cell death via causing mitochondrial damage. Although HIF- $1 \alpha$ is a transcription factor regulating BNIP3 expression ${ }^{49}$, we found in this study that silibinin treatment resulted in time-dependent downregulation of HIF- $1 \alpha$. This was also supported by previous study showing silibinin inhibited HIF-1 $\alpha$ expression in prostate cancer cells ${ }^{14}$. Oxidative stress was also reported to contribute to baicalein-induced BNIP3 upregulation in human osteosarcoma cells ${ }^{44}$. Consistently, we found as well that silibinin-induced BNIP3 upregulation and translocation to mitochondria were both apparently reversed when hydrogen peroxide was suppressed by supplement of GSH or autophagy was blocked. Given that silibinin-induced improvement of hydrogen peroxide was inhibited in the presence of 3MA or bafilomycin A1, we think that autophagy promoted silibinin-induced damage in mitochondria via regulation of BNIP3.

In conclusion, we demonstrated in this study that silibinin activates lethal autophagy though suppression of glycolysis. Autophagy improves intracellular hydrogen peroxide via depletion of GSH and cysteine by promoting p53 phosphorylation. Ultimately, hydrogen peroxide triggers BNIP3-dependnet mitochondria damages and nuclear translocation of AIF. Therefore, autophagy contributes to silibinin-induced glioma cell death via causing mitochondrial damage and nuclear translocation of AIF.

\section{Acknowledgements \\ This work was supported by National Nature and Science Foundation of China (81372697, 81772669, and 81972346), Changbaishan Scholar Project of Jilin province (2013026), Scientific Research Foundation of Jilin province (20150414013GH, 20200201405JC and 20190701051GH), and Foundation of the Health Department of Jilin province (2018Q026).}

\section{Author details \\ ${ }^{1}$ Department of Neurosurgery, First Hospital of Jilin University, 130021 Changchun, China. ${ }^{2}$ Research Center of Neuroscience, First Hospital of Jilin University, 130021 Changchun, China. ${ }^{3}$ Department of Radiotherapy, Second Hospital of Jilin University, 130021 Changchun, China. ${ }^{4}$ Department of Anesthesiology, First Hospital of Jilin University, 130021 Changchun, China. ${ }^{5}$ Department of Histology and Embryology, College of Basic Medical Sciences, Jilin University, 130021 Changchun, China. 'Key Laboratory of Pathobiology, Ministry of Education, Jilin University, 130021 Changchun, China}

\section{Author contributions}

This study was conceived, designed, and interpreted by G.P. and C.G. W.C., H.C., L.S., W.X., W.L., L.S., C.J., and P.M. undertook the data acquisition and analysis. C.G. and C.J. were responsible for the comprehensive technical support. G.P. and W.C. were major contributors in writing the manuscript. C.J. and C.G. contributed to the inspection of data and final manuscript. All authors read and approved the final manuscript.

\section{Data availability}

The data supporting the findings of this study are available within the article and supplementary files or from the authors upon reasonable request.

Conflict of interest

The authors declare that they have no conflict of interest.

\section{Publisher's note}

Springer Nature remains neutral with regard to jurisdictional claims in published maps and institutional affiliations. 
Supplementary Information accompanies this paper at (https://doi.org/ 10.1038/s41419-020-02866-3).

Received: 13 May 2020 Revised: 28 July 2020 Accepted: 29 July 2020 Published online: 14 August 2020

\section{References}

1. Lapointe, S., Perry, A. \& Butowski, N. A. Primary brain tumours in adults. Lancet 392, 432-446 (2018).

2. Messaoudi, K., Clavreul, A. \& Lagarce, F. Toward an effective strategy in glioblastoma treatment. Part I: resistance mechanisms and strategies to overcome resistance of glioblastoma to temozolomide. Drug Discov. Today 20, 899-905 (2015).

3. Yan, Y. et al. Targeting autophagy to sensitive glioma to temozolomide treatment. J. Exp. Clin. Cancer Res. 35, 23 (2016).

4. Levy, J., Towers, C. G. \& Thorburn, A. Targeting autophagy in cancer. Nat. Rev. Cancer 17, 528-542 (2017).

5. Awan, M. U. et al. Neuroprotective role of BNIP3 under oxidative stress through autophagy in neuroblastoma cells. Mol. Biol. Rep. 41, 5729-5734 (2014).

6. Su, Y. C. et al. Galectin-1-induced autophagy facilitates cisplatin resistance of hepatocellular carcinoma. PLoS ONE 11, e0148408 (2016).

7. Jiang, K. et al. Silibinin, a natural flavonoid, induces autophagy via ROSdependent mitochondrial dysfunction and loss of ATP involving BNIP3 in human MCF7 breast cancer cells. Oncol. Rep. 33, 2711-2718 (2015).

8. Duan, W. J. et al. Silibinin activated ROS-p38-NF-KB positive feedback and induced autophagic death in human fibrosarcoma HT1080 cells. J. Asian Nat. Prod. Res. 13, 27-35 (2011)

9. Ney, P. A. Mitochondrial autophagy: origins, significance, and role of BNIP3 and NIX. Biochim. Biophys. Acta 1853, 2775-2783 (2015).

10. Dany, M. \& Ogretmen, B. Ceramide induced mitophagy and tumor suppression. Biochim. Biophys. Acta 1853, 2834-2845 (2015).

11. Meyer, N. et al. AT 101 induces early mitochondrial dysfunction and HMOX1 (heme oxygenase 1) to trigger mitophagic cell death in glioma cells. Autophagy 14, 1693-1709 (2018)

12. Hosseinabadi, T. et al. Silymarin antiproliferative and apoptotic effects: insights into its clinical impact in various types of cancer. Phytother. Res. 33, 2849-2861 (2019).

13. Abenavoli, L. et al. Milk thistle (Silybum marianum): a concise overview on its chemistry, pharmacological, and nutraceutical uses in liver diseases. Phytother. Res. 32, 2202-2213 (2018).

14. Jung, $H$. J. et al. Silibinin inhibits expression of HIF-1alpha through suppression of protein translation in prostate cancer cells. Biochem. Biophys. Res. Commun. 390, 71-76 (2009)

15. Jeong, J. C. et al. Silibinin induces apoptosis via calpain-dependent AIF nuclear translocation in U87MG human glioma cell death. J. Exp. Clin. Cancer Res. 30, 44 (2011).

16. Elhag, R., Mazzio, E. A. \& Soliman, K. F. The effect of silibinin in enhancing toxicity of temozolomide and etoposide in p53 and PTEN-mutated resistant glioma cell lines. Anticancer Res. 35, 1263-1269 (2015).

17. Son, Y. G. et al. Silibinin sensitizes human glioma cells to TRAlL-mediated apoptosis via DR5 up-regulation and down-regulation of c-FLIP and survivin. Cancer Res. 67, 8274-8284 (2007).

18. Gülden, M. et al. Chrysin and silibinin sensitize human glioblastoma cells for arsenic trioxide. Food Chem. Toxicol. 105, 486-497 (2017).

19. Yang, Z. et al. Silibinin restores the sensitivity of cisplatin and taxol in A2780resistant cell and reduces drug-induced hepatotoxicity. Cancer Manag Res. 11, 7111-7122 (2019).

20. Lu, B. et al. RIP1 and RIP3 contribute to shikonin-induced glycolysis suppression in glioma cells via increase of intracellular hydrogen peroxide. Cancer Lett. 425, 31-42 (2018)

21. Imai, H., Matsuoka, M., Kumagai, T., Sakamoto, T. \& Koumura, T. Lipid peroxidation-dependent cell death regulated by GPx4 and ferroptosis. Curr. Top. Microbiol. Immunol. 403, 143-170 (2017).

22. Fu, J., Shao, C. J., Chen, F. R., Ng, H. K. \& Chen, Z. P. Autophagy induced by valproic acid is associated with oxidative stress in glioma cell lines. NeuroOncol. 12, 328-340 (2010).
23. Li, F. et al. Autophagy induction by silibinin positively contributes to its antimetastatic capacity via AMPK/mTOR pathway in renal cell carcinoma. Int. J. Mol. Sci. 16, 8415-8429 (2015).

24. Bai, Z. L., Tay, V., Guo, S. Z., Ren, J. \& Shu, M. G. Silibinin induced human glioblastoma cell apoptosis concomitant with autophagy through simultaneous inhibition of mTOR and YAP. Biomed. Res. Int. 2018, 6165192 (2018).

25. Zhou, B. et al. Ferroptosis is a type of autophagy-dependent cell death. Semin. Cancer Biol. S1044-579X, 30006-30009 (2019).

26. Goodall, M. L. et al. The autophagy machinery controls cell death switching between apoptosis and necroptosis. Dev. Cell 37, 337-349 (2016).

27. Zhang, Q. et al. Hexokinase II inhibitor, 3-BrPA induced autophagy by stimulating ROS formation in human breast cancer cells. Genes Cancer $\mathbf{5}$, 100-112 (2014).

28. Dey, P. et al. PKM2 knockdown induces autophagic cell death via AKT/mTOR pathway in human prostate cancer cells. Cell. Physiol. Biochem. 52, 1535-1552 (2019).

29. Shukla, S. K. et al. Silibinin-mediated metabolic reprogramming attenuates pancreatic cancer-induced cachexia and tumor growth. Oncotarget 6, 41146-41161 (2015)

30. $\mathrm{Yu}$, L. et al. Autophagic programmed cell death by selective catalase degradation. Proc. Natl Acad. Sci. USA 103, 4952-4957 (2006).

31. Jiao, L. et al. Regulation of glycolytic metabolism by autophagy in liver cancer involves selective autophagic degradation of HK2 (hexokinase 2). Autophagy 14, 671-684 (2018).

32. Wu, R. et al. Hexokinase $\|$ knockdown results in exaggerated cardiac hypertrophy via increased ROS production. EMBO Mol. Med. 4, 633-646 (2012).

33. Ham, J., Lim, W., Bazer, F. W. \& Song, G. Silibinin stimluates apoptosis by inducing generation of ROS and ER stress in human choriocarcinoma cells. J. Cell. Physiol. 233, 1638-1649 (2018).

34. Fan, S. et al. P53 activation plays a crucial role in silibinin induced ROS generation via PUMA and JNK. Free Radic. Res. 46, 310-319 (2012).

35. Fan, S. et al. P53-mediated GSH depletion enhanced the cytotoxicity of NO in silibinin-treated human cervical carcinoma HeLa cells. Free Radic. Res. 46, 1082-1092 (2012)

36. Horikawa, I. et al. Autophagic degradation of the inhibitory p53 isoform $\triangle 133 p 53 a$ as a regulatory mechanism for p53-mediated senescence. Nat. Commun. 5, 4706 (2014).

37. Ni, H. M., Williams, J. A. \& Ding, W. X. Mitochondrial dynamics and mitochondrial quality control. Redox Biol. 4, 6-13 (2015).

38. Fan, W. H. et al. Proteasome inhibitor MG-132 induces C6 glioma cell apoptosis via oxidative stress. Acta Pharmacol. Sin. 32, 619-625 (2011).

39. Ding, $Y$. et al. MLKL contributes to shikonin-induced glioma cell necroptosis via promotion of chromatinolysis. Cancer Lett. 467, 58-71 (2019).

40. Zheng, L. et al. JNK activation contributes to oxidative stress-induced parthanatos in glioma cells via increase of intracellular ROS production. Mol. Neurobiol. 54, 3492-3505 (2017).

41. Artus, C. et al. AlF promotes chromatinolysis and caspase-independent programmed necrosis by interacting with histone H2AX. EMBO J. 29, 1585-1599 (2010).

42. Candé, C. et al. AIF and cyclophilin A cooperate in apoptosis-associated chromatinolysis. Oncogene 23, 1514-1521 (2004).

43. Du, Y. et al. MicroRNA-145 induces apoptosis of glioma cells by targeting BNIP3 and Notch signaling. Oncotarget 8, 61510-61527 (2017).

44. Ye, F. et al. Baicalein induces human osteosarcoma cell line MG-63 apoptosis via ROS-induced BNIP3 expression. Tumour Biol. 36, 4731-4740 (2015).

45. Dhingra, R. et al. Bnip3 mediates doxorubicin-induced cardiac myocyte necrosis and mortality through changes in mitochondrial signaling. Proc. Natl Acad. Sci. USA 111, E5537-5544 (2014).

46. Shi, L. et al. MiR-21 protected human glioblastoma U87MG cells from chemotherapeutic drug temozolomide induced apoptosis by decreasing Bax/BCl2 ratio and caspase-3 activity. Brain Res. 1352, 255-264 (2010).

47. Stojcheva, N. et al. MicroRNA-138 promotes acquired alkylator resistance in glioblastoma by targeting the BCl-2-interacting mediator BIM. Oncotarget 7, 12937-12950 (2016).

48. He, Y. et al. Regulation of integrated stress response sensitizes U87MG glioblastoma cells to temozolomide through the mitochondrial apoptosis pathway. Anat. Rec. (Hoboken) 301, 1390-1397 (2018).

49. Niecknig, H. et al. Role of reactive oxygen species in the regulation of HIF-1 by prolyl hydroxylase 2 under mild hypoxia. Free Radic. Res. 46, 705-717 (2012). 